\title{
Sustainable technology results for sewage networks in smart cities
}

\author{
Antoni Grau, Yolanda Bolea \\ Technical University of Catalonia \\ Automatic Control Department \\ Barcelona, Spain \\ antoni.grau@upc.edu
}

\author{
Ana-Puig Pey, Alberto Sanfeliu \\ Robotics Industrial Institute \\ CSIC \\ Barcelona, Spain \\ Alberto.sanfeliu@iri.upc.edu
}

\author{
Josep Casanovas \\ Technical University of Catalonia \\ Statistics Department \\ Barcelona, Spain \\ josep.casanovas@upc.edu
}

\begin{abstract}
The objective of this paper is to explain the importance of research in wastewater transportation (sewage systems) using new technologies such as robotics systems and information and communication technologies. ECHORD++ (European Coordination Hub for Open Robotics Development) is a very useful tool to foster this research and to meet needs and solutions. In this paper, authors explain the tool as well as the methodology to promote robotics research in urban environments, and the on-going experience will demonstrate that huge advances are made in this field.
\end{abstract}

Keywords—Smart cities, Sustainability, Robotics, Communication systems, Sewer Systems.

\section{INTRODUCTION}

From their origins, cities have been the center of economic, political, urbanistic, cultural and social innovation fed by growing flows of people seeking work and business opportunities and, finally, better life conditions. The growth of the cities in a ordered and planned way generated multiple challenges to the people that live, work or visit the city, but specially to those who manage and administrate it. The improvement in management efficiency of public services, the environment quality (air, drinking water, public spaces for activities), people and goods mobility (logistics), attraction for economic activities, and general opinion of citizens in taking decisions have today a fundamental ally: information and communication technologies and, more recently, robotics [1]. The concept of Smart City is neither unique nor universal. But there is a common concept: the use of Information and Communication Technology ICT seeking at the same time and holistically the more featured developments in the digital transformation of cities towards the sustainability, efficiency and citizens and visitors' wellness, [2].

Europe has a long tradition of outstanding research and manufacturing in robotics. However, finding common ground between manufacturers and the research community has proven difficult in the past. Defining the future direction of robotics research has revealed to be the real challenge. European Coordination Hub for Open Robotics Development, ECHORD++ [3], was installed as an incubator to promote innovation by facilitating the cooperation between academia and industry. ECHORD++ wants to further stimulate this interaction between robot manufacturers, researchers and users. This goal will be achieved by implementing three different instruments: i) the experiments, ii) Public and users Driven Technological Innovation (PDTIs) and iii) Robotic and Innovation Facilities (RIFs). Specifically, project managers will focus in PDTI, that it is the methodology followed to develop a final technological product that is innovative and ready for commercialization (throughout the pairing academia-industry). Another key feature is that the product is not yet in the market and it is expected to be the solution for more than one cities.

Regarding the PDTI side, ECHORD++ consists of different stages: initially, local authorities in city councils analyze the existing problems in the city that should be solved in a technological manner (by means of a robotized solution); each city can present a unique proposal in a public call; secondly, when all the presented proposals are collected a panel of experts score and rank them following some specific indicators (citizen's needs, social impact, sustainability impact, economic impact, efficiency...), only the best ranked proposal wins and this proposal will be "the challenge" to be solved; then, the proposal is refined by the winner city and presented, a public call is open to consortia (research centers and private companies) as a European project and only the two best ranked solutions are accepted to be working to solve the challenge. These two consortia are funded by ECHORD++ project to work in parallel for solving the challenge (Phase I, Phase II and Phase III, see Fig.1), [4]. At the end of this process of 36 months, the suitable prototypes (evaluated by panel of experts- final reviewers) will be considered for industrial production and deployment.

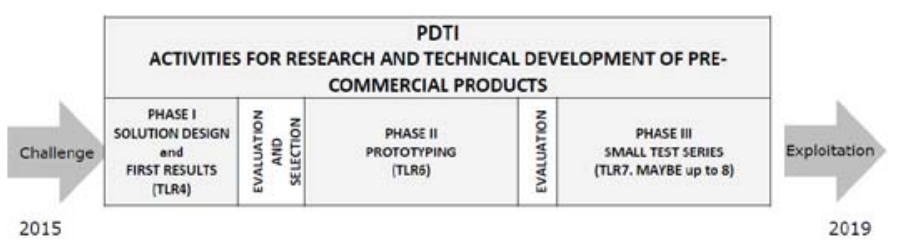

Fig. 1. PDTIs activities for research and technical development of commercial products 


\section{The PHASES Of THE ECHORD++ ProJeCT}

\section{A. The Process}

After a selection among 14 European cities that submit an innovative technological challenge, the city of Barcelona won the challenge in Urban Robotics with the project proposal "Robots for the inspection and the clearance of the sewer network in cities". After this selection an open call was performed by ECHORD++ administration and a total of 10 consortiums applied for the project. Finally, two consortiums were selected to develop their research in this challenge, ending up with a real prototype which will be presented at the end of the 36-month project. Some specific European regulations exist regarding this subject (Regulation (EC) No 1013/2006, and Decision No 1386/2013/EU). To develop this research project, proposers have to follow the Barcelona city regulations in this matter, Environmental Municipal Ordinance (1999). The new robotics solutions should include: 1) mobility and autonomy and 2) communication and teleoperation (ICTs). At the end of the project, the innovative technological solutions for the challenge of the Barcelona city proposed by both consortiums will be evaluated by a panel of experts, and it is expected that two robotics commercial products are the outcome of the project,

\section{B. The challenge}

Sewer inspections require many people to work in risky and unhealthy conditions. Introducing a robotic solution in this process aims at reducing the labour risks, improving the precision of sewer inspections and optimizing sewer cleaning resources of the city. This system should be able to determine the state of the sewer in order to identify sewer segments where its functionality has been reduced either by sediments or by structural defects. Other functionalities required are sewer monitoring and water, air and sediment sampling. To well carry out these tasks, some general functions are required like remote operation, video and images capture, scanning and map building, communication and teleoperation, among others. Those items have to be approached with efficiency and in a sustainable manner. As we mentioned in the Introduction, two consortiums (SIAR, Sewer Inspection Autonomous Robot and ARSI, Aerial Robot for Sewer Inspection) have currently developing two possible technological solutions.

\section{New Technology and objectives}

The requirements for the new technology are given by the inherent sewer characteristics, that is, different ranges of pipe sizes, possible high concentration of, not explosive, but toxic gases as hydrogen sulphide, slippery areas, obstacles, atmosphere with $100 \%$ humidity, water temperature $16{ }^{\circ} \mathrm{C}$, and no telecommunication coverage in the sewer. The devices that could be considered as robots are able to move themselves in one direction by sewer and record video in $360^{\circ}$ to register the state of the sewer, they have to analyse the sewer by zooming and navigating in $360^{\circ}$ by the video images, they need to access into the sewer system at one point and being recuperated in other point in an autonomous way, they are equipped with cameras, Laser, Lidar and Inertial navigation systems, Sonar sensor (for underwater detection if there is some stream of water) and hydrogen sulphide sensor, using a powerful communication system. With all data collected by the sensors, it is possible to generate a model of the interior of the sewer and identify the possible impairments. The improvements in the existing technology that this project seeks are to facilitate realtime decision making, innovation that makes inspection devices more autonomous, to have more degrees of freedom to move around the network, and the possibility to intensify the checking of a zone where impairment has been detected.

The objective of developing this new technology is to mechanize sewer inspections in order to reduce the labour risks, objectify sewer inspections and optimize sewer cleaning expenses of the city. Regarding the sewer monitoring, the objective of sewer monitoring is to approximate the robot to the maximum level of sensitivity which will allow the sewer manager to make decisions without exposing to risky locations. The task of sample collecting is greatly important in order to obtain valid and traceable information which could be used afterwards to determine environmental legislation and policies.

\section{Description of the pilot case: Barcelona sewer system}

The current need of the City of Barcelona is to mechanize sewer inspections in order to reduce the labour risks, objectify sewer inspections and optimize sewer cleaning expenses of the city. The sewer network of Barcelona is $1,532 \mathrm{~km}$ long, from which approximately $50 \%$ is accessible, which means that the pipe is at least $1.5 \mathrm{~m}$ high and workers are allowed to go inside it. In order to determine the state of the network, visual inspections are done with different frequencies depending on the slope and other characteristics of the sewer. Workers walk all along the pipe, in some sections even four times a year, and decide where it is necessary to clean. Moreover, sewers are classified as confined spaces which require special health and safety measures, in addition to other risks like slippery sections, obstacles or biological risks from the eventual contact with wastewater. These features made the process of sewer inspection a risky and expensive process that requires improvements urgently. Sewer inspection is a service included in the public management of the sewers of Barcelona. Nowadays, sewer inspections are done by people performing visual inspections and collecting information about the state of the sewage like sediment level and type, pipe obstructions, etc. Because of the sewer risks, the performance of the inspections is about $1.5 \mathrm{~km}$ of sewer every 6 hours. There are two technological solutions proposed (SIAR and ARSI projects) that are currently working and making progresses. Phase I of both projects has successfully showed the proposed solution of each one as proof of concept in the tests conducted in mobility, autonomy and communications. Currently these projects are in Phase II, during which all the aspects of the solution will be developed and validated to ensure a complete solution for robot-assisted sewer inspection. It is expected to obtain a prototype inspecting the Barcelona sewer network at the end this phase. 


\section{SIAR PROPOSAL}

The SIAR solution seeks to solve the problem by the creation of an autonomous robot (see, Fig.2 (Left), with the possibility of control from a human operator in case of need of emergency. By using the proposed wireless system, augmented by the deployment of self-powered wireless repeaters, it will be possible to transmit bidirectional data between robot and an external operator, allowing the operator to change the mission in real time, instead of having to wait for the end of the sewer exploration to identify problems on the sewer system. The SIAR system will go beyond existing solutions through the inclusion of some innovative features, while maintaining affordable costs: configurable locomotion system with interchangeable wheels and tracks modules and variable length/width of the traction system; reliable navigation system based on data fusion from low-cost commercial RGB-D cameras, IMU and encoders, performing accurate localization and navigation on the sewer system.
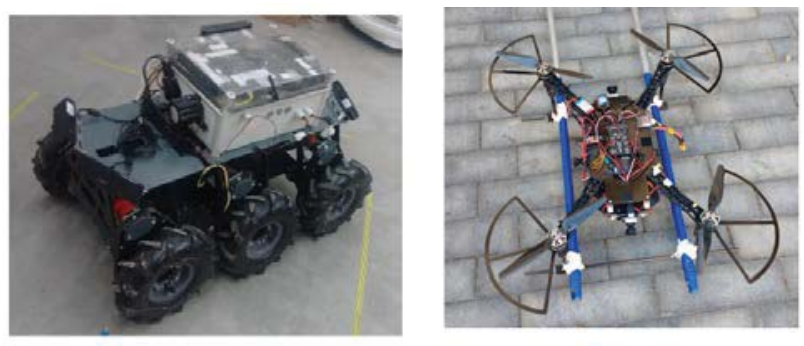

Fig. 2. (Left) SIAR prototype of Phase 1. (Right) ARSI platform setup

The SIAR project will develop a fully autonomous ground robot able to navigate through the sewage system with a minimal human intervention and with the possibility of manually controlling the vehicle or the sensor payload when required.

Communications rely on line of sight. As long as this line of sight is maintained the range and performance is satisfactory. The consortium intends to use repeaters to maintain communication beyond line of sight. It is recommended that the consortium evaluates the practicalities of deployment and retrieval of the repeaters in more detail as they may become a major impediment to practical application. Following the comments of the review, the consortium has been working (in Phase I) on the following aspects to analyze and improve the practicalities of the communication repeaters deployment/retrieval tasks: 1) Final design of the repeater package, including weight, batteries, autonomy and functionalities. 2) Design of the arm, including DoF (degrees of freedom), sizes, gripper and maximum payload. 3) Sensing approaches for estimating the relative position between robot and repeater for picking up the repeaters back to the robot (passive and active markers). The communication block will be equipped with a long range $2.4 \mathrm{GHz}$ router, the Microhard nVIP2400. This device has been tested indoors and proved to communicate up to 300 meters with line of sight. This link will provide a high bandwidth connection that could be used for video streaming and additional information such as the $3 \mathrm{D}$ reconstruction of the environment. With the proposed battery the repeater power autonomy will be close to 4 hours. Please note that this consumption is calculated in the worst case scenario (repeater working at its maximum consumption and also the Arduino platform at full load) and letting the batteries to be discharged at $80 \%$ of their capacity, following the manufacturer's guidelines. To increase this autonomy, the repeater will be equipped with a small microcontroller system that can communicate with the robot through a Bluetooth module. This module will allow us to disconnect the nVIP2400 device when not deployed to save batteries, to measure the state of the batteries and to send this information to the base station. In this way, it is expected that each repeater will have power enough for performing one day of operation (to be confirmed during field trials). The deployment of repeaters whenever the Line-of-Sight (LoS) is expected to be lost, and therefore the quality of the links could begin to degrade, is proposed. This will extend the operational range of the robot in more than 300 $\mathrm{m}$. for each repeater. To this end, the robotic platform will be equipped with a 4DoF manipulator on its back, which will automatically pick-up one of the available communication repeaters and deploy it on the ground (see Fig.3). This manipulator will also be used to retrieve the already deployed repeaters in its way back.

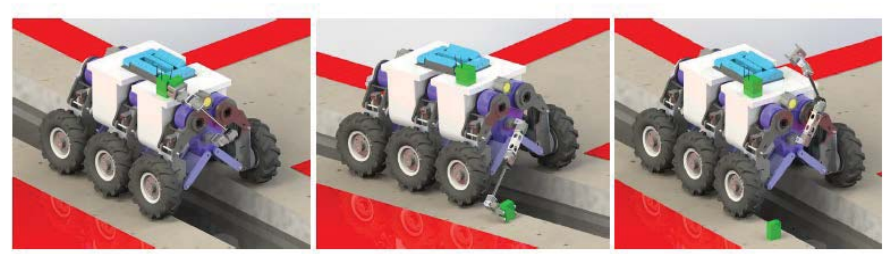

Fig. 3. SIAR Robot deploying one wifi communication repeater.

\section{ARSI PROPOSAL}

The proposal is the use of a Micro Aerial Vehicle (MAV) (see Fig.2(Right)) for inspection tasks in the sewer avoids the mobility constraints from which a ground robot would suffer, such as paths with steps, steep drops and even objects in the way. Additionally, a flying platform is able to move faster through the sewer network than the terrestrial alternative and needs simpler logistics in deployment and operation. On the other hand, a MAV solution has to overcome strong constraints of size, weight and energy, as its flying space is bounded by sections less than $100 \mathrm{~cm}$ wide. Therefore, its size and consequently payload are limited to minimal dimensions. The ARSI consortium has addressed the problem of sewer inspection with the integral design of an aerial platform, multi-rotor type, endowed with sensors for semi-autonomous navigation and data collection within network, and capable of communicating with an on-surface operator. The different drones that will operating inside the sewer will be monitored and controlled from a van parked outside, in the street, and the workers will assist the robots to replace the batteries [5].

This section describes how the problem of the communications between the ARSI aerial vehicle and the 
Remote Station in the sewer was addressed and solved. Firstly, the challenge of the problem is described. As a result of a study of the problem and of the available technologies, the solution is described. The procedure aims at optimizing the range provided by our communications system and the autonomy of the batteries. To provide radio coverage in tunnel-like environments (tunnels, mines), two main methods are used: the leaky feeder, based on the use of radiating cables, and systems based on the natural propagation of radio-waves. Owing to the high cost of leaky-feeder installations and the fact that they are susceptible to damage and failure, the natural propagation system is preferred in many applications. Wireless propagation in these environments is described as strongly multipath, and if the wavelength of the signal is much smaller than the tunnel cross section, tunnels act as an oversized dielectric waveguide. In this case, the attenuation per unit length is low enough to allow communications over a range of up to several kilometers.

However, the signal is affected by strong fading phenomena, as has been studied by many authors [6]. The environment that a sewer creates for telecommunications is challenging to say the least. Wired communications are not feasible given the aerial nature of our platform. Moreover, the multiple ground obstacles (corners, changes of level, water, etc.) present in sewers result in wired communications being impractical for almost any type of mobile platform. In the domain of wireless communications, only very low frequency signal travels without impediment in the underground. However, only high frequency waves provide the bandwidth and link performance necessary to meet the video and data streaming requirements of this project.

ARSI consortium experimental results have confirmed that sewer tunnels act as a waveguide for Wi-Fi signal. In straight stretches of tunnels, Wi-Fi signal travels for hundreds of meters with very little attenuation. In these experiments we paid close attention to the attenuation at bifurcations in the sewer network. While sharp turns (90 degrees or more) result in a nearly complete loss of Wi-Fi signal, we observed that shallow turns also act as waveguides and that Wi-Fi signal keeps propagating for large distances after. This is a crucial observation because the topology of sewer networks is specifically designed to merge sewage water flows and direct them towards evacuation points. This topology is shown clearly in Fig.4 which depicts a section of the Barcelona sewer network (in green) in the Eixample area where the ARSI evaluation will take place.

The key to our methodology is to position our Wi-Fi emitter at locations where the generated signal range will be maximal based on the topology of the network. In Figure 40, the red line models which sewer tunnels would be within Wi-Fi coverage given the location of the emitter. We can see that from this location, the Wi-Fi signal travelling south only encounters bifurcations with sharp angles, therefore none of the tunnels in the side streets (Corsega and Rosselló in this example) would be within Wi-Fi range for an inspection using the ARSI platform. More specifically, the project pretends to achieve: 1) Reliable Wi-Fi propagation with Line of Sight ( $\mathrm{LoS})$ in sewer tunnels of lengths between 100-150m, typical in Barcelona, including in the Phase II evaluation area around Mercado del Born. 2) Build a model of the Wi-Fi propagation around curves and bends in the tunnels. This characteristic of sewer networks, which we observed during Phase I, gives users more flexibility when planning inspections using the ARSI platform. 3) Since the kickoff meeting in mid-November 2016, one visit to the sewers around Mercat del Born have been carried out, primarily to get to know their specificities and potential difficulties for the aerial platform. The next milestone is to carry out extensive communications tests in Mercat del Born in 2017.

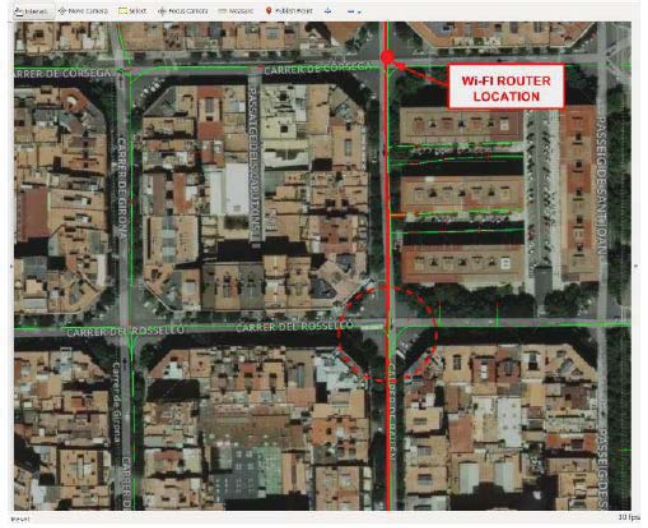

Fig. 4. Wi-Fi propagation (in red) in Barcelona serwer tunnels (i

\section{CONCLUSIONS}

In this paper, the challenge of sewage cleaning monitoring using robotics and communications is shown. This challenge is carried out by two selected consortiums in the ECHORD++. The communication solution proposed in Phase I for both consortiums is explained obtaining satisfactory results. Next, in Phase II, a prototype (from the communication solution proposed en Phase I) will be presented. Then, both consortiums once again will be reviewed to pass Phase III.

\section{ACKNOWLEDGMENT}

These research has been funded by the European project "ECHORD++ EU project PF7-ICT-2012-601116".

\section{REFERENCES}

[1] Ontiveros, E., Vizcaíno, D. and López Sabater, V., Las ciudades del futuro: inteligentes, digitales y sostenibles; Telefonica Fundación, 2016, in Spanish.

[2] Smart Cities World, https://smartcitiesworld.net/news/news/rise-of-therobots-879, accessed on Oct $6^{\text {th }} 2017$

[3] ECHORD++ website, http://echord.eu/, accessed on Oct $6^{\text {th }} 2017$.

[4] A. Puig-Pey, Y. Bolea, A. Grau, and J. Casanovas, "Public entities driven robotic innovation in urban areas", Robotics and autonomous systems, Vol. 92, pp. 162-172, 2017.

[5] Flying robots in sewers. "El Pais" newspaper, December $7^{\text {th }}, 2015$, https://elpais.com/ccaa/2015/12/06/catalunya/1449424748_228383.html

[6] D.G. Dudley, M. Lienard, S.F. Mahmoud, and P. Degauque. "Wireless propagation in tunnels" Antennas and Propagation Magazine, IEEE, vol. 49, no. 2, pages 11-26, April 2007. 